\title{
Simple Methods for Enhancing Patient Outcome in Routine Care: Measuring, Monitoring, and Feedback
}

\author{
Michael J. Lambert ${ }^{1 \bowtie}$ \& Gianluca Lo Coco ${ }^{2}$
}

\begin{abstract}
While highly effective, psychotherapy outcome studies suggest 5-14\% of clients worsen while in treatment and that therapists are unable to identify a substantial portion of such cases. Methods to systematically track client mental health functioning over the course of treatment and adjust treatment through the use of problem-solving tools are described. We summarize meta-analyses of the effects of a feedback system indicating that the number of psychotherapy patients who deteriorate can be cut in half. We conclude with a series of practice implications, including that clinicians seriously consider making formal methods of collecting client feedback a routine part of their daily practice.
\end{abstract}

Keywords: client feedback, Outcome Questionnaire-45, treatment failure

Reviews of psychotherapy research, both qualitative and quantitative, have shown that about $75 \%$ of those who enter treatment show benefit (Lambert, 2013). An often ignored but critical consideration in psychotherapy is the degree to which they have negative rather than positive consequences for clients. An estimated $5-10 \%$ of adult clients participating in clinical trials leave treatment worse off than they began treatment (Lambert \& Ogles, 2004).

In routine care the situation is more problematic. Outcomes for more than 6,000 patients treated in routine practice settings suggest that the clients did not fare as well as those in clinical trials, with only about one-third showing improvement or recovery (Hansen, Lambert, \& Forman, 2002). The situation for child psychotherapy in routine care is even more sobering. The small body of outcome studies in communitybased, usual care settings has yielded a mean effect size near zero (e.g., Weisz, 2004), yet millions of youth are served each year in these systems of care. In a comparison of children being treated in community mental health $(N=936)$ or through managed care $(N=3075)$ estimates of deterioration were $24 \%$ and $14 \%$, respectively (Warren et al., 2010).

There is no doubt that all of the deterioration that

\footnotetext{
${ }^{1}$ Brigham Young University, Provo, UT, USA.

${ }^{2}$ University of Palermo, Italy.

$\triangle$ Correspondence concerning this paper should be addressed to Department of Psychology, Brigham Young University, Provo, UT, USA 84602. E-mail: michael_lambert@byu.edu
}

occurs during the time a patient is in treatment cannot be causally linked to therapist activities. Certainly, a portion of patients are on a negative trajectory at the time they enter treatment and the deteriorating course cannot be stopped even if a portion of patients are prevented from taking their own lives or do not otherwise improve. Just as positive psychotherapy outcomes depend largely on patient characteristics, so do the negative changes that occur in patients who are undergoing psychological treatments. Even so, positive as well as negative patient change can be affected by therapist actions and inactions. Research reviews find that the major contribution of the therapist to negative change is usually found in the nature of the therapeutic relationship, with rejections of either a subtle or manifest nature being the root cause (e.g., Lambert, Bergin, \& Collins, 1977; Safran, Muran, Samstang, \& Winston, 2005).

\section{The problem}

Unfortunately, clinicians appear to have an overly optimistic view of their patients' progress estimating $85 \%$ of their clients improve and that they are superior to their peers in achieving positive outcomes (Walfish et al., 2012). This high estimate can be contrasted with the portion of clients who improve in formally studied psychotherapy, where the estimate of positive patient outcome is closer to two thirds. Clinicians also appear to overlook negative changes and have a limited capacity to make accurate predictions of the final benefit clients will receive during treatment, particularly with 
clients who are failing to improve. One study, for example, found that even when therapists were provided with the base rate of deterioration in the clinic where they worked (8\%), and were asked to rate each client that they saw at the end of each session (with regards to the likelihood of treatment failure and if the patient was worse off at the current session in relation to their intake level of functioning), they rated only 3 of 550 clients as predicted failures and seriously underestimated worse functioning for a significant portion of clients (Hannan et al., 2005). A recent study (Chapman et al., 2012) suggested that therapist underestimate the number of clients who deteriorate during group therapy and were unable to predict client perceptions of the group relationship. A retrospective review of case notes of clients who had deteriorated during treatment found infrequent mention of worsening even when its degree was dramatic (Hatfield, McCullough, Plucinski, \& Krieger, 2010).

Such results are not surprising, given psychotherapist optimism, the complexity of persons, and a treatment context that calls for considerable commitment and determination on the part of the therapist, who actually has very little control over the patient's life circumstances and personal characteristics. Patients' response to treatment is, especially in the case of a worsening state, a likely place where outside feedback might have the greatest chance of impact. Helping the therapist become aware of negative change and discussing such progress in the therapeutic encounter are much more likely when formal feedback is provided to therapists. Such feedback helps the client to communicate and helps the therapist to become aware of the possible need to adjust treatment, alter or address problematic aspects of the treatment as appropriate (e.g., problems in the therapeutic relationship or in the implementation of the goals of the treatment).

In a meta-analysis of the effects of informing clinicians of the changing health status of their patients, Sapyta (quoted in Sapyta, Riemer, \& Bickman, 2005) examined 30 randomized clinical trials conducted in community settings that assessed the effectiveness of client health status feedback to health professionals. The nature of feedback interventions and methods of their delivery varied from giving general practice physicians depression or anxiety screening information about their patients to repeatedly and routinely providing clinicians with their patients' mental health status feedback. The typical client in the feedback group was better off than $58 \%$ of the control group $(d=.21$, a small effect).

The Sapyta and colleagues (2005) review indicated that the effectiveness of feedback is likely to vary as a function of the degree of discrepancy between therapists views of progress and measured progress, and that the greater the discrepancy the more likely it is that feedback will be helpful. A key element of effective feedback is bringing into the recipient's awareness the discrepancy between what is thought and what is "reality," thereby prompting corrective action.
In general, this research supports the conclusion that feedback in clinical practice improves patient outcome.

This finding is consistent with feedback theories that suggest feedback will only change behavior when the information provided indicates the individual is not coming up to an established standard (e.g., Riemer \& Bickman, 2004). Riemer and Bickman have developed a Contextual Feedback Intervention Theory to explain how feedback is interpreted and made useful (Riemer \& Bickman, 2004; Riemer, Rosof-Williams, \& Bickman, 2005). Basic tenets of this theory are that clinicians (and professionals, generally) will benefit from feedback if they are committed to the goal of improving their performance, aware of a discrepancy between the goal and reality (particularly if the goal is attractive and the clinician believes it can be accomplished), the feedback source is credible, feedback is immediate, frequent, systematic, cognitively simple (such as graphic in nature) and unambiguous, and provides clinicians with concrete suggestions of how to improve.

If clinicians do not regard feedback as credible, valid, informative, or useful, they are more likely to dismiss it whenever it does not fit their own preferences. As we know from research on cognitive dissonance, people can change attitudes rather than persevering toward goals, thus regarding the goal as less important, or see a client as too resistant or injured to benefit from treatment (e.g., disown personal responsibility for meeting the goal of positive functioning; Riemer, Rosof-Williams, \& Bickman, 2005). As feedback research suggests, the value of monitoring and systematic feedback through psychological assessments hinges on the degree to which the information provided goes beyond what a clinician can observe and understand about patient progress without such information. It is important for the information to add something to the psychotherapist's view of patient well-being and future actions.

A recent trend in clinical practice involves regularly monitoring and tracking client treatment response with standardized scales throughout the course of treatment and providing clinicians with this information before the client leaves treatment. The basic rationale behind collecting client feedback is based on common sense. If we get information about which patients are improving during treatment and which are not our responsiveness to clients will improve. In many situations, performance and feedback are intertwined and obvious; in others, a certain degree of blinding occurs, meaning the temporal link is not clear and the effects of performance are harder to discern (such as in psychotherapy), making it much more difficult for the therapist to learn and improve. In obvious as well as more subtle situations, providing feedback to improve performance has been studied quite extensively in a variety of areas and confirms our common sense expectations that it is helpful.

In this article, we define treatment monitoring, feedback, and problem-solving strategies used specifically in psychotherapy. We then present some meta- 
analytic findings to understand the impact of such methods before turning our attention to their implications for clinical practice.

\section{Definitions and feedback measures}

Clients can complete a brief measurement of their psychological functioning by using standardized rating scales and then this information can be delivered to psychotherapists in real time. This feedback alerts therapists to deviations from expected treatment response and thereby provides novel information to therapists and clients. Collecting this information from the client on a session-by-session basis provides the clinician with a systematic way of monitoring life functioning from the client's point of view. A brief formal assessment can provide a summary of life functioning that is not otherwise available to the therapist, unless the therapist spends time within the treatment hour to systematically inquire about all the areas of functioning covered by the self-report scale, an activity that is time consuming and detracts from service delivery.

\section{OQ Psychotherapy Quality Management System}

Lambert and colleagues developed the OQ system, which emphasizes the measurement of mental health functioning and includes a measure of the therapistclient relationship, social supports, motivation, and untoward life events. The Outcome Questionnaire- 45 (OQ-45; Lambert et al., 2004) is a 45-item, self-report measure designed for repeated administration throughout the course of treatment and at termination with adult patients. This measure is based on Lambert's (1983) conceptualization suggesting that three aspects of the client's life should be monitored: (a) subjective discomfort (intrapsychic functioning; e.g., "I feel blue," "I have thoughts of ending my life"), (b) interpersonal relationships (e.g., "I feel lonely," "my sex life is satisfactory"), and (c) social role performance (e.g., "I feel stressed at work/school," "I am afraid I might do something at work/school that I might regret"). Items on this instrument address commonly occurring problems across a wide variety of disorders and tap the symptoms most likely to occur. The items also measure personally and socially relevant characteristics that affect the individual's quality of life. Each item is scored on a 5-point scale (0 $=$ never, $1=$ rarely; $2=$ sometimes; $3=$ frequently; $4=$ almost always), yielding a range of possible scores of 0 to 180 with higher values indicating greater levels of psychological disturbance. The respondent is asked to answer with respect to how they have been feeling during the past week. Consistent with this conceptualization of outcome, the OQ- 45 provides a Total Score, based on all 45 items, as well as Symptom Distress, Interpersonal Relations, and Social Role subscale scores. Each of these subscales contains some items related to the positive quality of life of the individual.
Research has indicated that the OQ- 45 is a psychometrically sound instrument, with strong internal consistency, adequate test-retest reliability and strong concurrent validity (Lambert et al., 2004). Furthermore, the items that make up the OQ- 45 have been shown to be sensitive to changes in multiple client populations over short periods of time while remaining relatively stable in untreated individuals (Vermeersch et al., 2004). Evidence from factor analytic studies suggests it measures an overall psychological distress factor as well as factors consistent with the three subscales (e.g., de Jong et al., 2007; Lo Coco et al., 2008). It provides clinicians with a mental health vital sign. Similar measures have been developed for use with children (www.oqmeasures.com).

A core element of the OQ system is the prediction of treatment failure. In order to improve outcomes of clients who are responding poorly to treatment, such clients must be identified before termination, and ideally, as early as possible in the course of treatment. The OQ system plots a statistically-generated expected recovery curve for differing levels of pretreatment distress and uses this as a basis for identifying clients who are not making expected treatment gains and are at risk of having a poor outcome (noton-track cases). The accuracy of this signal-alarm system has been evaluated in a number of empirical investigations (e.g., Ellsworth, Lambert, \& Johnson, 2006; Lambert et al., 2002). A sample feedback report for the OQ-45 is available at the OQ Measures web site previous noted.

In conjunction with identifying Alarm status, an instrument, Assessment for Signal Cases (ASC; Lambert et al., 2007) was developed to assist clinicians to problem-solve with the clients who backslide during treatment (i.e., when a therapist receives a warning message predicting deterioration). This 40 -item measure does not produce a total score, but rather provides subscale score feedback and item feedback for therapists to consider in problem-solving. The first 11items of the ASC require the client to reflect on the therapeutic relationship and report his or her perceptions. The ASC is central to the Clinical Support Tool (CST; Lambert et al., 2007; Lambert Whipple, Harmon, et al., 2004) which is composed of a problemsolving decision tree designed to systematically direct therapists' attention to subscales and items: the therapeutic alliance, social support, readiness to change, diagnostic formulation, life events and need for medication referral.

\section{Review of effects}

The OQ system was designed to enhance the outcome of clients predicted to experience treatment failure at termination. Accordingly, the studies examining the effects of the OQ systems conducted separate analyses for at-risk clients and on track clients (Shimokawa, Lambert, \& Smart, 2010). Because different effect size units were employed in original studies, units of 
Table 1. Characteristics of the studies used in the meta-analyses

\begin{tabular}{lccc}
\hline Clients/ & Age Mean & \\
Study & Therapists & (SD) & Key findings \\
\hline
\end{tabular}

Lambert, Whipple, (2001)

Lambert, Whipple, Vermeersch et al. (2002)

Whipple et al. (2003)

Hawkins et al. (2004)

Harmon et al. (2007) Smart et al.

$609 / 36 \quad 22.23(3.92)$

Participants in the study were clients who received treatment in a university counseling center. They were randomly assigned to the therapist feedback condition or the no therapist feedback condition. Patients of both conditions improved over the course of therapy. Regarding patients who were not progressing (not on track), those in the feedback condition reported lower OQ scores at termination than the patients in the no feedback condition. Statistically significant effects of the feedback intervention were also found in keeping not-ontrack (NOT) patients in treatment longer than patients in the no feedback condition.

1020/56 22.26 (3.65) Participants in the study were clients who received treatment in a university counseling center. They were randomly assigned to the therapist feedback condition or the no therapist feedback condition. Results showed that feedback to therapist improved the client's outcome at the end of treatment, and improved outcome for clients identified as potential treatment failures (NOT). Moreover, 31.9\% of NOT-therapist feedback clients reported a reliable or clinically significant change, compared to $17.7 \%$ in the NOT no-feedback condition.

981/48 22.88 (3.54) Participants in the study were clients who received treatment in a university counseling center. They were randomly assigned to the therapist feedback and the no-feedback condition. When a client was identified as not-on-track (NOT), therapists had the option to use the Clinical Support Tools (CST), which included a decision tree and CST measures. To determine whether the use of CST in addition to feedback improved the outcome of NOT clients, three groups were compared: the feedback plus Clinical Support Tools (CST) condition, the therapist feedback condition, and the no-feedback condition. Clients in the CST plus feedback group showed larger treatment gains ( $49 \%$ showed a reliable or clinically significant change) than clients in the therapist feedback (33\%) and in the no-feedback condition (25\%). Moreover, in the CST plus feedback condition, significantly fewer patients met criteria for deterioration at termination.

$201 / 5 \quad 30.8(10.5)$

Participants in the study were outpatients seeking treatment at a hospital-based clinic. They were randomly assigned to one of three conditions: therapist feedback group, patient-therapist feedback group, and no feedback to therapist. Clients in the patient/therapist feedback condition reported larger treatment outcome over both therapist feedback and no feedback conditions. Regarding patients in each of the three treatment groups that were not on track, the proportion of patients categorized as improved (i.e., met the either reliable or clinically significant change criteria) at the end of treatment was .32 , .40, and .56 for the no feedback, therapist feedback and patient/therapist feedback conditions, respectively.

1374/72 22.68 (3.68) Participants in the study were clients seeking treatment at a university counseling center. Participants were randomly assigned to therapist feedback group and patient-therapist feedback group. These two groups were compared to an archival control group (receive no feedback). All three groups improved conditions based on their OQ-45 scores from pretreatment to post-treatment. The improvement in symptoms, however, was greater for the two treatment groups compared to the archival control group, $\mathrm{p}<.001$. Additional analyses were conducted among patients in each of the two treatment groups that were not progressing (i.e. not on track). Patients classified as not on track were randomized into CST feedback or no CST feedback. The feedback treatment plus CST group had fewer patients that deteriorated $(7.4 \%)$ compared to 17.95 in the feedback treatment group, and $21.3 \%$ no feedback archival group. The feedback treatment plus CST had $42.1 \%$ had reliable clinical improvement, compared to $29.2 \%$ of patients from the feedback treatment group, and $21 \%$ from the no feedback archival group.

Slade et al. $\quad 1101 / 73 \quad 24.25$ (3.29) The research design essentially replicated the study design of the Harmon et al. study, except (2008) for the use of the OQ-Analyst (IEF), a computer software that provided immediate, electronic progress feedback. Clients attending treatment at a university counseling center were randomly assigned to one of two treatment conditions: a therapist feedback condition and a client/therapist feedback condition. Archival TAU control groups from existing feedback study data sets were also used. Results indicated significant pre to post improvement for each feedback group. In addition, clients who were NOT were randomly divided into two groups: therapist CST feedback and no therapist CST feedback. Results indicated that clients in the progress feedback plus CST feedback condition reported greater improvement than their no CST feedback counterparts. Regarding feedback timing, results of the study suggested that delaying the CST use by a session had no effect on outcome. Finally, the use of the OQ- 45 IEF plus the CST feedback tripled (63.9\% vs $21 \%)$ the number of clients who were rated as recovered/reliably improved. 
effect size were applied. For each comparison of mean post-treatment outcome scores between an experimental condition and a treatment as usual (TAU) control, standardized differences in means $(d)$ were calculated, using pooled standard deviations (notated as $g$ in Hedges, 1981) and transformed $d$ effect sizes into correlation $r$, utilizing a commonly used formula of $r=d /$ sqr $\left(d^{2}+4\right)$ (e.g., Wolf, 1986). When comparing results from controlled trials, the results were aggregated meta-analytically to obtain weighted effect sizes, employing a random effects model (Hedges \& Vevea, 1998). When comparing the feedback treatments in relation to an archival control group, effect sizes were obtained from a combined dataset pooled across studies, employing a mega-analytic approach to obtain the $d$ statistic (Shimokawa et al., 2010).

A key element in psychotherapy research is operationalizing the concepts of positive and negative outcome for the individual client. Jacobson and Truax (1991) offered a methodology by which individual client changes on an outcome measure can be classified in the following categories: recovered, reliably improved, no change, deteriorated. There are two necessary pieces of information to make client's outcome classifications: a Reliable Change Index (RCI) and a normal functioning cut off score. Clinical and normative data were analyzed by Lambert and colleagues (2004) to establish a Reliable Change Index (RCI) and a cut off score for the OQ-45. To contrast the rates and odds of client deterioration and significant improvement between feedback groups and TAU, Shimokawa and colleagues (2010) calculated combined odds ratios $(O R)$ as a measure of effect size. Specifically, when examining the odds of deterioration, they dichotomized clients into either the deterioration group or non-deterioration group and calculated the odds ratio of deterioration for a given comparison. Similarly, when comparing the odds of improvement in two groups, the odds ratio was calculated based on the odds of improvement versus the odds of nonimprovement.

In the Shimokawa and colleagues (2010) review of the OQ system, data were re-analyzed $(N=6,151)$ from all six OQ feedback studies published to that date (Harmon et al., 2007; Hawkins, Lambert, Vermeersch, Slade, \& Tuttle, 2004; Lambert, Whipple, et al., 2001; Lambert, Whipple, Vermeersch, et al., 2002; Slade, Lambert, Harmon, Smart, \& Bailey, 2008; Whipple et al., 2003). Each of the studies evaluated the effects of providing feedback about each client's improvement through the use of progress graphs and warnings about clients who were not demonstrating expected treatment responses (not-on-track cases). The six studies shared many design and methodological features (see Table 1): (a) Consecutive cases seen in routine care regardless of client diagnosis or comorbid conditions (rather than being disorder specific); (b) Random assignment of clients to experimental conditions (various feedback interventions) and TAU conditions (no feedback) was made in four of the six studies, while reasonable measures were taken in two studies to ensure equivalence in experimental and control conditions at pre-treatment; (c) Psychotherapists provided a variety of theoretically guided treatments, with most adhering to cognitive behavioral and eclectic orientations and fewer representing psychodynamic and experiential orientations; (d) A variety of therapist experience-post-graduate therapists and graduate students each accounted for about 50\% of clients seen; (e) Therapists saw both experimental (feedback) and no feedback cases, thus limiting the likelihood that outcome differences between conditions could be due to therapist effects; (f) The outcome measure as well as the methodology rules/standards for identifying signal-alarm clients (failing cases) remained constant; (g) The length of therapy (dosage) was determined by client and therapist rather than by research design or arbitrary insurance limits. The meta-analysis (Shimokawa et al., 2010) involved both intent-to-treat (ITT) and efficacy analyses on the effects of various feedback interventions in relation to TAU. In the analysis presented here (See Table 2) only those clients who received and completed the treatments were compared to treatment-as-usual (TAU).

Effects of $O Q$ progress feedback $(F b)$ on noton-track clients. Not-on-track clients made up 11 to $33 \%$ of the total sample. When the not-on-track $\mathrm{Fb}$ group was compared to the not-on-track TAU group, the effect size for post-treatment OQ score difference averaged $r=.25$ ( $g=.53), 95 \%$ CI $[.15, .34], p<.001$. These results suggest that the average at risk client whose therapist received feedback was better off than approximately $70 \%$ of at risk clients in the no feedback condition (routine care). In terms of the clinical significance at termination, $9 \%$ of those receiving feedback deteriorated while $38 \%$ achieved clinically significant improvement. In contrast, among at risk clients whose therapists did not receive feedback, 20\% deteriorated while $22 \%$ showed clinically significant improvement. When the odds of deterioration and clinically significant improvement were compared, results indicated those in the feedback group had less than half the odds of experiencing deterioration while having approximately 2.6 times higher odds of experiencing reliable improvement. To be consistent in effect size unit, the odds ratios were converted to correlation $r$ and presented in Table 2.

Effects of patient/therapist feedback ( $P / \mathbf{T ~ F b})$ on not-on-track clients. The effect size of posttreatment $\mathrm{OQ}$ score, based on a mega-analysis on pooled datasets of the $\mathrm{P} / \mathrm{T} \mathrm{Fb}$ group and the TAU, was $r=.25(g=.55),[.17, .33], p<.001$-effects very similar to that of the therapist only feedback group $(\mathrm{Fb})$. The rates of deterioration and clinically significant improvement when both participants received feedback were $15 \%$ and $45 \%$, respectively. The results suggest that clients who received feedback along with their therapist had approximately .7 times the odds of deterioration, while having approximately three times higher odds of achieving clinically significant improve- 
Table 2. Effect sizes of client feedback in comparison to TAU

\begin{tabular}{|c|c|c|c|c|c|}
\hline \multirow[b]{2}{*}{ Feedback system } & \multirow[b]{2}{*}{$k$} & \multirow[b]{2}{*}{$\begin{array}{l}\operatorname{Exp} n / \\
\text { TAU } n\end{array}$} & \multicolumn{3}{|c|}{$r[95 \% \mathrm{CI}]$} \\
\hline & & & $\begin{array}{l}\text { Post-treatment } \\
\text { score }\end{array}$ & $\begin{array}{c}\text { Reliable } \\
\text { improvement }\end{array}$ & Deterioration $^{\mathrm{a}}$ \\
\hline \multicolumn{6}{|l|}{ OQ System ${ }^{\mathrm{b}}$} \\
\hline $\mathrm{NOT} \mathrm{Fb}$ & 4 & $136 / 318$ & $0.25^{* * *}[.15, .34]$ & $0.23^{* * *}[.13, .32]$ & $-0.21^{*} \quad[-.35,-.05]$ \\
\hline NOT P/T Fb & 3 & $177 / 318$ & $0.25^{* * *}[.17, .33]$ & $0.27^{* * *}[.17, .36]$ & $-0.10 \quad[-.23, \quad .03]$ \\
\hline $\mathrm{CST} \mathrm{Fb}$ & 3 & $217 / 318$ & $0.33^{* * *}[.25, .40]$ & $0.34^{* * *}[.26, .42]$ & $-0.37^{* * *}[-.50, .22]$ \\
\hline
\end{tabular}

Note. $k=$ number of studies; Exp = experimental group; TAU = treatment as usual group; $r=$ effect size expressed in correlation $r$; $\mathrm{CI}=$ Confidence Interval; NOT $\mathrm{Fb}=$ not-on-track clients whose therapists received client progress feedback; NOT $\mathrm{P} / \mathrm{T} \mathrm{Fb}=$ not-on-track clients where both clients and therapists received client progress feedback. $\mathrm{CST}$ Fb $=$ not-on-track clients whose therapists received client progress feedback and Clinical Support Tools feedback.

${ }^{a}$ Negative correlations indicate greater effect in reducing treatment failure at termination.

' Effect sizes (Hedges' $g$ and $O R$ ) of the OQ system-based feedback interventions were meta- and mega-analytically calculated and reported in Shimokaw and colleagues (2010). Effect sizes from the NOT Fb group represents weighted effect sizes based on a random effects model. NOT P/T Fb and CST Fb effect sizes were meta-analytically obtained from an aggregated data set pooled across studies.

${ }^{*} p<.05 .{ }^{* *} p<.01{ }^{* * *} p<.001$.

ment. These results suggest that, although the average client who received feedback along with their therapist was better off than about $71 \%$ of clients in TAU, there may have been moderators that facilitated outcome enhancement in some clients while failing to prevent, or possibly contributing to worsening.

Effects of Clinical Support Tools feedback (CST Fb) on not-on-track clients. When the outcome of clients whose therapist received the Clinical Support Tool feedback were compared to the treatment-as-usual clients, the effect size for the difference in mean post-treatment OQ scores was $r=.33(\mathrm{~g}=$ $.70), 95 \%$ CI $[.25, .40], p<.001$. These results indicate that the average client in the Clinical Support Tool feedback group, who stays in treatment to experience the benefit of this intervention, are better off than $76 \%$ of clients in treatment-as-usual. The rates of deterioration and clinically significant improvement among those receiving Clinical Support Tools were $6 \%$ and $53 \%$, respectively. The results suggest that clients whose therapists used Clinical Support Tools have less than a fourth the odds of deterioration, while having approximately 3.9 times higher odds of achieving clinically significant improvement.

\section{Limitations of the research}

Major limitations of feedback research are the small number of studies evaluating effectiveness, the limited number of researchers responsible for the findings reviewed here, and the sole reliance on self-report measures. Since the mega/meta-analysis reported here on the OQ-Analyst three more recent studies have been conducted, one with substance abusing patients (Crits-Christoph et al., 2012); one with eating disordered patients treated in an inpatient hospital (Simon et al., 2013); and one in a hospital-based outpatient setting (Simon et al., 2012). Findings from these stud- ies suggest that the effects of feedback improve outcomes over those obtained by the same therapists practicing TAU (i.e., without the assistance of feedback and clinical support tools). Simon and colleagues (2013) found progress feedback with Clinical Support Tools compared to TAU resulted in 59\% versus $29 \%$ reaching statistically significant change. But the size of effects and the proportions of clients achieving clinically significant benefits were generally smaller than those achieved in the earlier studies. Such findings are difficult to interpret, but may be due to a greater degree of patient disturbance, less than once a week psychotherapy, or other factors. It also appears that feedback had effects on all patients, not just those at risk for treatment failure. It is likely that future research will be done across a wider range of treatment settings, countries, and patient populations, thus illuminating the limits of these procedures and clarifying the factors that maximize patient gains. Feedback is limited in settings where individuals feel it may be in their interest to understate (or overstate) their problems and produce inaccurate ratings of their mental health functioning. Feedback systems are predicated on accurate self-reporting of levels of disturbance and corresponding changes.

\section{Cross-cultural applications of the feedback system}

It is also worth noting that efforts to bridge the gap between research and practice by enhancing patient outcome before treatment termination and issues of quality assurance have started to be reported in most of European health care systems over the last few years. Appropriate methods to prevent patient's treatment failure have increased their feasibility in routine clinical care (Lutz et al., 2006) and were used as a screening method in clinical settings.

Many mental health care providers have adopted 
strategies to provide outcome monitoring feedback to clinicians and patients (i.e., the Clinical Outcomes in Routine Evaluation-Outcome Measure in the United Kingdom; see Evans et al., 2002). In Germany, for example, a large health insurance company decided to conduct a quality monitoring study in outpatient psychotherapy with therapists in private practice. Therapists with different orientation (e.g., CBT, psychodynamic, and psychoanalytic) in three regions of Germany agreed to participate in the study adopting the feedback system. This ongoing research project aims to investigate whether the effectiveness of psychotherapy can be enhanced by the use of psychometric quality monitoring self-reports, and increase the transparency of psychotherapeutic treatments by providing progress information to patients and supporting the therapists in adaptive decision making (Lutz et al., 2011).

One of the most interesting and comprehensive European research programs on monitoring progress and providing feedback has been conducted by the team coordinated by Kim de Jong in the Netherlands. To date, two studies have been conducted by adopting the OQ feedback system. The first (de Jong et al., 2012) included 413 outpatients recruited from two mental healthcare institutions, who were randomly assigned to the feedback group or control group. Despite finding no beneficial effect of feedback to therapists on the rate of change in patients for the full sample, in not-on-track cases a positive effect was found when therapists indicated that they used the feedback. It seems that feedback may not be effective under all circumstances for all therapists. Future research focusing on how therapists use the feedback and how it works is warranted.

A second study (de Jong, 2012) on the effect of feedback to therapists and patients included 110 psychotherapists with different theoretical orientations, who treated 475 outpatients in private practice or mental health care institutions. Subjects were randomly assigned to one of three conditions: feedback to therapist, feedback to both therapist and patient, and a control group without feedback. Interestingly, the effect of feedback was studied in both short-term and long-term therapies. It was found that feedback to both therapists and patients was most effective. The benefits were strongest for patients who were not improving well in short-term therapies. A small but significant effect of the feedback to both therapists and patients on rate of change was found in long-term therapies. These findings raise the question of why feedback seemed most effective for NOT cases in short-term therapies but not in long-term therapies. As suggested by the authors, maybe in longer treatments therapists have more opportunities to identify and correct the negative trajectory of patients, or maybe patients who receive long-term therapies are not the same type of patients who receive short-term treatments. As previously mentioned, further research is needed across a wider range of treatment modalities (i.e., short-term/long-term), and patient populations (i.e., level of disturbance).

To date in Italy the (perceived) practical difficulties of adding monitoring activities to busy practices has been an important barrier to implementation of feedback systems. Italian practitioners often express skepticism in relation to the additional expenditure of time because of data management issues. We hope that the development of the Italian version of the OQ-45 (Chiappelli et al., 2008; Lo Coco et al., 2006), the translation of the Clinical Support Tools (Lo Coco et al., 2003) as well as recent advances in software programs may make the possibility of feedback to clinicians easy to implement.

Two core issues could make formal outcome monitoring with feedback suitable for the Italian public mental health care system: First, research has already demonstrated that when feedback was provided, patient's progress was achieved faster, which may result in more cost-effective interventions; second, the feedback is most effective with NOT cases who have an increased risk of achieving negative treatment outcomes (Lutz et al., 2006); so, the outcome monitoring system may reduce patients' drop-out rates and development of chronic conditions.

We hope that the results of the feedback studies convinces practitioners that systematically monitoring their patients with the methods described here is in the best interest of patients and that researchers will consider replication in Italian clinical settings.

\section{Summary}

The use of real-time client feedback to monitor patients' response to psychotherapy and satisfaction with the therapy relationship improves psychotherapy outcomes and especially does so for clients at risk for deterioration. Feedback probably works because it compensates for therapists' limited ability to accurately detect client worsening in psychotherapy. Despite considerable evidence that psychotherapists are not alert to treatment failure (e.g., Hannan et al., 2005; Hatfield et al., 2010), and strong evidence that clinical judgments are usually inferior to actuarial methods, therapists' confidence in their clinical judgment stands as a barrier to implementation of monitoring and feedback systems. It appears to be helpful to supplement progress feedback with clinical support tools. As suggested by the general literature on feedback and the evidence presented here, problem-solving and decisionenhancement tools prove helpful to clinicians and, most importantly, clients whose treatment response is in doubt. As yet we are uncertain of the necessity of sharing progress feedback directly with clients.

\section{References}

Chapman, C. L., Burlingame, G. M., Gleave, R., Rees, F., Beecher, M., \& Porter, G. S. (2012). Clinical prediction in group psychotherapy. Psychotherapy Research, 22(6), 673-681. doi: 10.1080/10503307.2012.702512

Chiappelli, M., Lo Coco, G., Gullo, S., Bensi, L., \& Prestano, C. 
(2008). L'Outcome-Questionnaire 45.2. Adattamento italiano di uno strumento per la valutazione dei trattamenti psicologici [Outcome-Questionnaire 45.2: Italian version of a measure for evaluating the psychological treatment]. Epidemiologia e Psichiatria Sociale, 17(4) 152-161. doi: $10.1037 / \mathrm{a} 0022426$

Crits-Christoph, P., Ring-Kurtz, S., Hamilton, J., Lambert, M. J., Gallop, R., McClure, B., ... Rotrosen, J. A. (2012). Preliminary study of the effects of individual patient-level feedback in outpatient substance abuse treatment programs. Journal of Substance Abuse Treatment, 42(3), 301-309._doi: 10.1016/j.jsat.2011.09.003

de Jong, K. (2012). A chance for change. Building an outcome monitoring feedback system for outpatient mental health care. Dissertation Leiden University. Ridderprint Grafisch Bedrijf, Ridderkerk.

de Jong, K., Nugter, M. A., Polak, M. G., Wagenborg, J. E. A., Spinhoven, P., \& Heiser, W. J. (2007). The Outcome Questionnaire (OQ-45) in a Dutch population: a cross-cultural validation. Clinical Psychology and Psychotherapy, 17(4), 288301. doi: 10.1002/cpp.529

de Jong, K., Van Sluis, P., Nugter, M.A., Heiser, W.J., \& Spinhoven, P. (2012). Understanding the differential impact of outcome monitoring: therapist variables that moderate feedback effects in a randomized clinical trial. Psychotherapy Research, 22(4), 464-474. doi: 10.1080/10503307.2012.67

Ellsworth, J. R., Lambert, M. J., \& Johnson, J. (2006). A comparison of the Outcome Questionnaire- 45 and Outcome Questionnaire-30 in classification and prediction of treatment outcome. Clinical Psychology and Psychotherapy, 13(6), 380-391. doi: 10.1002/cpp.503

Evans, C. Connell, J., Barkham, M., Margison, F., McGrath, G., \& Mellor-Clark, J. (2002). Towards a standardized brief outcome measure: psychometric properties and utility of the CORE-OM. The British Journal of Psychiatry, 180, 51-60.

Hannan, C., Lambert, M. J., Harmon, C., Nielsen, S. L., Smart, D. W., Shimokawa, K., \& Sutton, S. W. (2005). A lab test and algorithms for identifying clients at risk for treatment failure. Journal of Clinical Psychology: In Session, 61(2), 15563. doi: $10.1002 /$ jclp.20108

Hansen, N. B., Lambert, M. J., \& Forman, E. V. (2002). The psychotherapy dose-response effect and its implications for treatment delivery services. Clinical Psychology: Science and Practice, 9, 329-343.

Harmon, S. C., Lambert, M. J., Smart, D. W., Hawkins, E. J., Nielsen, S. L., Slade, K., \& Lutz, W. (2007). Enhancing outcome for potential treatment failures: Therapist/client feedback and clinical support tools. Psychotherapy Research, 17(4), 379-392. doi:10.1080/10503300600702331

Hatfield, D., McCullough, L., Plucinski, A., \& Krieger, K. (2010). Do we know when our clients get worse? An investigation of therapists' ability to detect negative client change. Clinical Psychology \& Psychotherapy, 17(1), 25-32. doi: 10.1002/cpp.656

Hawkins, E. J., Lambert, M. J., Vermeersch, D. A., Slade, K., \& Tuttle, K. (2004). The effects of providing patient progress information to therapists and patients. Psychotherapy Research, 14(3), 308-327. doi: 10.1093/ptr/kph027.

Hedges, L. V. (1981). Distribution theory for Glass's estimator of effect size and related estimators. Journal of Educational Statistics, 6, 107-128. doi: 10.2307/1164588

Hedges, L. V. \& Vevea, J. L. (1998). Fixed- and random-effects models in meta-analysis. Psychological Methods, 3(4), 486504. doi: 10.1037/1082-989X.3.4.486

Jacobson, N. S., \& Truax, P. (1991). Clinical significance: A statistical approach to defining meaningful change in psychotherapy research. Journal of Consulting and Clinical Psychology, 59(1), 12-19.

Lambert, M. J. (1983). Introduction to assessment in psycho- therapy outcome: Historical perspective and current issues, In M. J. Lambert, S.S. DeJulio \& E. R. Christensen, (Eds.), The Assessment of Psychotherapy Outcome. New York: Wiley-Interscience.

Lambert, M. J. (2013). The efficacy and effectiveness of psychotherapy. In M. J. Lambert (Ed.), Bergin and Garfield's handbook of psychotherapy and behavior change ( $6^{\text {th }}$ ed., pp. 139-193). New York: Wiley.

Lambert, M. J., Bailey, R. J., Kimball, K., Shimokawa, K., Harmon, S. C., \& Slade, K. (2007). Clinical Support Tools Manual-BriefVersion-40. Salt Lake City: OQMeasures LLC.

Lambert, M. J., Bergin, A. E., \& Collins, J. L. (1977). Therapist induced deterioration in psychotherapy patients (pp. 452481). In A. S. Gurman, \& A. M. Razin (Eds.), Effective psychotherapy: $A$ handbook of research. New York: Pergamon Press.

Lambert, M. J., Morton, J. J., Hatfield, D., Harmon, C., Hamilton, S., Reid, R. C., ... Burlingame, G. M. (2004). Administration and Scoring Manual for the Outcome Questionnaire -45. SLC, UT: OQ Measures.

Lambert, M. J., \& Ogles, B. M. (2004). The efficacy and effectiveness of psychotherapy. In M. J. Lambert (Eds.) Bergin \& Garfield's Handbook of Psychotherapy and Behavior Change (5 $5^{\text {th }}$ ed., pp. 139-193). New York: Wiley.

Lambert, M. J., Whipple, J. L., Bishop, M. J., Vermeersch, D. A., Gray, G. V., \& Finch, A. E. (2002). Comparison of empirically derived and rationally derived methods for identifying patients at risk for treatment failure. Clinical Psychology and Psychotherapy, 9(2), 149-164. doi: 10.1007/s10826-0055049-1

Lambert, M. J., Whipple, J. L., Harmon, C., Shimokawa, K., Slade, K., \& Christofferson, C. (2004). Clinical Support Tools Manual, Department of Psychology, Brigham Young University, Provo UT.

Lambert, M. J., Whipple, J. L., Smart, D. W., Vermeersch, D. A., Nielsen, S. L., \& Hawkins, E. J. (2001). The effects of providing therapists with feedback on client progress during psychotherapy: Are outcomes enhanced? Psychotherapy Research, 11(1), 49-68.

Lambert, M. J., Whipple, J. L., Vermeersch, D. A., Smart, D. W., Hawkins, E. J., Nielsen, S. L., \& Goates, M. K. (2002). Enhancing psychotherapy outcomes via providing feedback on client progress: A replication. Clinical Psychology and Psychotherapy, 9(1), 91-103. doi:10.1002/cpp.324

Lo Coco, G., Chiappelli, M., Bensi, L. Gullo, S., Prestano, C., \& Lambert, M. J. (2008). The factorial structure of the Outcome Questionnaire-45: A study with an Italian sample. Clinical Psychology \& Psychotherapy, 15, 418-423. doi: $10.1002 / \mathrm{cpp} .601$

Lo Coco G., Prestano C., Di Stefano G., Gullo S., \& Lambert M. J . (2006). Un primo studio sulla validazione italiana dell'Outcome Questionnaire (OQ-45.2). [A first study about the Italian adaptation of the Outcome Questionnaire (OQ45.2)]. Ricerca in Psicoterapia, 9(2), 7-19.

Lo Coco G., Prestano C., Lambert M. J. (2003). La ricerca focalizzata sul paziente. Un modello di supporto clinico per il terapeuta. [Research focused on the patient. A model of clinical support for the therapist]. Ricerca in Psicoterapia, 6(3), 135-149.

Lutz, W., Saunders, S. M., Leon, S. C., Martinovich, Z., Kosfelder, J., Schulte, D., ... Tholen, S., (2006). Empirically and clinically useful decision making in psychotherapy: Differential predictions with treatment response models. Psychological Assessment, 18(2), 133-141.doi: 10.1037/10403590.18.2.133

Lutz, W., Bohnke, J. R., \& Kock, K. (2011). Lending an ear to feedback systems: evaluation of recovery and non response in psychotherapy in a German outpatient setting. Community Mental Health Journal, 47(3), 311-317. doi:10.1007/s10597- 
010-93073

Riemer M., \& Bickman L. (2004) The Contextualized Feedback Intervention Theory: $A$ Theory of Guided Behavior Change. Nashville, TN: Unpublished Manuscript.

Riemer, M., Rosof-Williams, J., \& Bickman, L. (2005). Theories related to changing clinician practice. Child Adolescent Psychiatric Clinics of North America, 14(12), 241-254.

Safran, J. D., Muran, J. C., Samstang, L. W. \& Winston, A. (2005). Evaluating alliance-focused intervention for potential treatment failures: A feasibility and descriptive analysis. Psychotherapy: Theory, Research, Practice, \& Training, 42, 512-531.

Sapyta, J., Riemer, M, \& Bickman, L. (2005). Feedback to clinicians: Theory, research, and practice. Journal of Clinical Psychology, 62(2), 145-153.

Shimokawa, K., Lambert, M. J., \& Smart, D. W. (2010). Enhancing treatment outcome of patients at risk of treatment failure: Meta-analytic and mega-analytic review of a psychotherapy quality assurance system. Journal of Consulting \& Clinical Psychology, 78(3), 298-311. doi:10.1037/a00192247

Simon, W., Lambert, M. J., Busath, G., Vazquez, A., Berkeljon, A., Hyer, K., Granley, M., \& Berrett, M. (2013). Effects of providing patient progress feedback and clinical support tools to psychotherapists in an inpatient eating disorders treatment program: A randomized controlled study. Psychotherapy Research, 23(3), 287-300. doi:: 10.1080/10503307.2013.787497

Simon, W., Lambert, M. J., Harris, M. W., Busath, G., \& Vazquez, A. (2012). Providing patient progress information and clinical support tools to therapists: Effects on patients at risk for treatment failure. Psychotherapy Research, 22(6), 638647. doi: 10.1080/10503307.2012.698918

Slade, K., Lambert, M. J., Harmon, S. C., Smart, D. W., \& Bailey, R. (2008). Improving psychotherapy outcome: The use of immediate electronic feedback and revised clinical sup- port tools. Clinical Psychology \& Psychotherapy, 15,_287-303. doi: $10.1002 /$ cpp.594

Vermeersch, D. A., Whipple, J. L., Lambert, M. J., Hawkins, E. J., Burchfield, C. M., \& Okiishi, J. C. (2004). Outcome Questionnaire: Is it sensitive to changes in counseling center clients? Journal of Counseling Psychology, 51(1), 38-49. doi: 10.1037/0022-0167.51.1.38

Walfish, S., McAlister, B., O’Donnell, P., \& Lambert, M. J. (2012). An investigation of self- assessment bias in mental health providers. Psychological Reports, 110(2), 639-644. doi: 10.2466/02.07.17

Warren, J. S., Nelson, P. L., Mondragon, S. A., Baldwin, S. A., \& Burlingame, G. M. (2010). Youth psychotherapy change trajectories \& outcome in usual care: Community mental health versus managed care. Journal of Clinical \& Consulting Psychology, 78(11), 144-155. doi: 10.1037/a0018544

Weisz, J. R. (2004). Psychotherapy for Children and Adolescents: Evidence-Based Treatments and Case Examples. New York, NY: Cambridge University Press.

Whipple, J. L., Lambert, M. J., Vermeersch, D. A., Smart, D. W., Nielsen, S. L., \& Hawkins, E. J. (2003). Improving the effects of psychotherapy: The use of early identification of treatment failure and problem solving strategies in routine practice. Journal of Counseling Psychology, 58(1), 59-68. doi:10.1037/0022-0167.50.1.59

Wolf, F. M. (1986). Meta-analysis: Quantitative methods for research synthesis. Sage University Paper series on quantitative applications in the social sciences. Beverly Hills: Sage Publications.

Received April 17, 2013

Revision September 11, 2013

Accepted October 16, 2013 